\title{
The Value of Ultrasonography in the Diagnosis and Monitoring of Localized Morphea - Case Report
}

\author{
Anca Chiriac ${ }^{1,2}$, Piotr Brzezinski ${ }^{3}$, Anca E. Chiriac ${ }^{4}$, Marius Florin Coroș ${ }^{5}$, Cosmin Moldovan $^{6}$, \\ Cristian Podoleanu 7 , Simona Stolnicu ${ }^{8}$ \\ 1 Department of Dermatology, Nicolina Medical Center, Iași, Romania \\ 2 Department of Dermato-Physiology, Apollonia University, Iași, Romania \\ ${ }^{3}$ Department of Dermatology, $6^{\text {th }}$ Military Support Unit, Poland \\ 4 "Gr. T. Popa" University of Medicine and Pharmacy, Iași, Romania \\ 5 Department of Surgery, University of Medicine and Pharmacy, Tîrgu Mureș, Romania \\ 6 Department of Histology, University of Medicine and Pharmacy, Tîrgu Mureș, Romania \\ 7 Department of Internal Medicine IV, University of Medicine and Pharmacy, Tîrgu Mureș, Romania \\ 8 Department of Pathology, University of Medicine and Pharmacy, Tîrgu Mureș, Romania
}

\section{CORRESPONDENCE}

Cristian Podoleanu

Str. Gheorghe Marinescu nr. 38

540139 Tîrgu Mureș, Romania

Tel +40 265215551

E-mail: podoleanu@me.com

\section{ARTICLE HISTORY}

Received: 23 August, 2016

Accepted: 9 September, 2016
Anca Chiriac • Str. Hatman Sendrea nr. 2, 700613 lași, Romania. Tel: +40 232251725

Piotr Brzezinski $\cdot 6^{\text {th }}$ Military Support Unit, os. Ledowo $1 \mathrm{~N}, 76-270$ Ustka, Poland

Anca E. Chiriac • Str. Muzicii nr. 2, 700399 lași, Romania. Tel +40 232210310

Marius Florin Coros • Str. Gheorghe Marinescu nr. 38 540139 Tîrgu Mureș, Romania. Tel +40 265215551

Cosmin Moldovan • Str. Gheorghe Marinescu nr. 38 540139 Tîrgu Mureș, Romania. Tel +40 265215551

Simona Stolnicu • Str. Gheorghe Marinescu nr. 38 540139 Tîrgu Mures, Romania. Tel +40 265215551

\begin{abstract}
Introduction: The aim of this presentation is to highlight the usefulness of high-frequency ultrasound (18 MHz) in localized morphea for: identification of the lesion, guided skin biopsy, quantification of skin thickness, evaluating the severity by measuring total echogenicity. Case presentation: A 62-year-old Caucasian woman was referred to the Dermatology Department for a well-circumscribed indurate plaque localized on the right side of the abdominal wall and thigh. On clinical examination, a large well-delimited, indurate plaque, silvery in the center and surrounded by a purplish-red halo (lilac ring) was noticed on the right side of the abdomen and thigh. An ultrasound-guided punch biopsy was carried out and the microscopic examination of the biopsy revealed moderate interstitial inflammatory infiltrate together with abundant collagen bundles in the dermis and subcutis and a diagnosis of localized morphea (scleroderma) was established. Ultrasonography was performed and skin thickness was measured using high-frequency US (18 MHz) and was found to be $3.1 \mathrm{~mm}$ to $3.9 \mathrm{~mm}$. Conclusion: high frequency ultrasound is an inexpensive, easy to perform, noninvasive method, replacing surgical biopsy and offering a valuable quantification of skin fibrosis.
\end{abstract}

Keywords: ultrasound, morphea, skin disease

\section{INTRODUCTION}

Localized morphea (or scleroderma) is characterized by excessive collagen deposition that leads to thickening of the dermis and/or subcutaneous tissues. This process can be demonstrated by clinical and histopathological examination of a skin biopsy from the affected area. Ultrasound imaging has been used in explor- 
ing various skin neoplasms, regional lymph node involvement by metastases, dermatomyositis, vascular anomalies, but its day-to-day use in dermatology is not well defined. ${ }^{1,2}$ In general, ultrasound may be of use when choosing the skin areas that would yield a positive biopsy specimen, for assessing the size and extension of the lesions and appropriate treatment. Ultrasound scan has been previously used in localized morphea for evaluating various sonographic parameters. It has been demonstrated by other studies to be a practical instrument in the identification of this type of lesion and evaluating the severity of the lesion by measuring total echogenicity, hypodermis echogenicity and deep tissue layer vascularity. ${ }^{3,4} \mathrm{~A}$ correlation between clinical severity index (erythema, warmth, violaceous color, new lesion, expansion of lesion, induration) and ultrasound findings was studied, comparing active and inactive lesions. ${ }^{4}$ The aim of this presentation is to highlight the usefulness of high-frequency (18 MHz) ultrasound exploration in localized morphea for quantitative assessment of skin thickness, virtual skin biopsy and monitoring the lesion.

\section{CASE PRESENTATION}

A 62-year-old Caucasian woman was referred to the Dermatology Department for a well-circumscribed indurate plaque localized on the right side of the abdominal wall and thigh (Figure 1). Her medical history included arterial hypertension. The patient described no other symptoms associated with the skin lesion, her unique medical concern was esthetic.

On clinical examination, a large, well-delimited, indurate plaque, silvery in the center and surrounded by a purplish-red halo (lilac ring) was noticed on the right side of the abdomen and thigh.

Thorough investigations were performed: chest X-ray and tomography with no pulmonary involvement; echocardiogram was reported as normal; renal function, C-reactive protein, bilirubin, GOT and GPT levels and urine analysis were normal; leukocytes count with normal hemoglobin and differential count; C3, C4, rheumatoid factor, Scl70, lupic anticoagulant, ANAs, anti DNA, antineutrophil cytoplasmic antibodies (ANCA), anticardiolipins, anti-Ro, anti-La, anti-Sm, anti-RNP 4.8 all within standard values; abdominal ultrasonography showed no pathological findings. Serologic screening for Borrelia burgdorferi, hepatitis virus $\mathrm{B}$ and $\mathrm{C}$ were negative. Thyroid function was normal.

An ultrasound-guided punch biopsy was carried out, and the microscopic examination of the biopsy revealed moderate interstitial inflammatory infiltrate together with abundant collagen bundles in the dermis and subcutis, and a diagnosis of localized morphea (scleroderma) was established. Ultrasonography was performed (Figure 2), and skin thickness was measured using high frequency US (18 MHz) and was found to be $3.1 \mathrm{~mm}$ to $3.9 \mathrm{~mm}$. The images were recorded for patient follow-up. Ultrasound scan was also performed on unaffected areas around the morphea plaque for comparison. A very potent steroid cream was recommended for daily use and a close clinical surveillance was asked.

Ultrasonography was performed monthly for the following 4 months after the diagnosis, measuring the skin thickness within the morphea plaque. A positive clinical outcome was demonstrated using ultrasound examination and by evaluating the ultrasound parameters, since skin thickness diminished to $1.8 \mathrm{~mm}$ after 4 months of topical steroid use.

\section{DISCUSSIONS}

Localized morphea is a lesion usually limited to the skin and subcutaneous tissue; on very rare occasions, it can involve the underlying bone, while in the craniofacial subtype, nervous system involvement has been described. ${ }^{5}$ Microscopically, excessive collagen deposition leads to thickening of the dermis and/or subcutaneous tissues. These alterations of the skin structures can be demonstrated by ultrasonography. Skin ultrasonography has been used previously occasionally in other types of skin lesions, but also in localized morphea as a tool in monitoring the

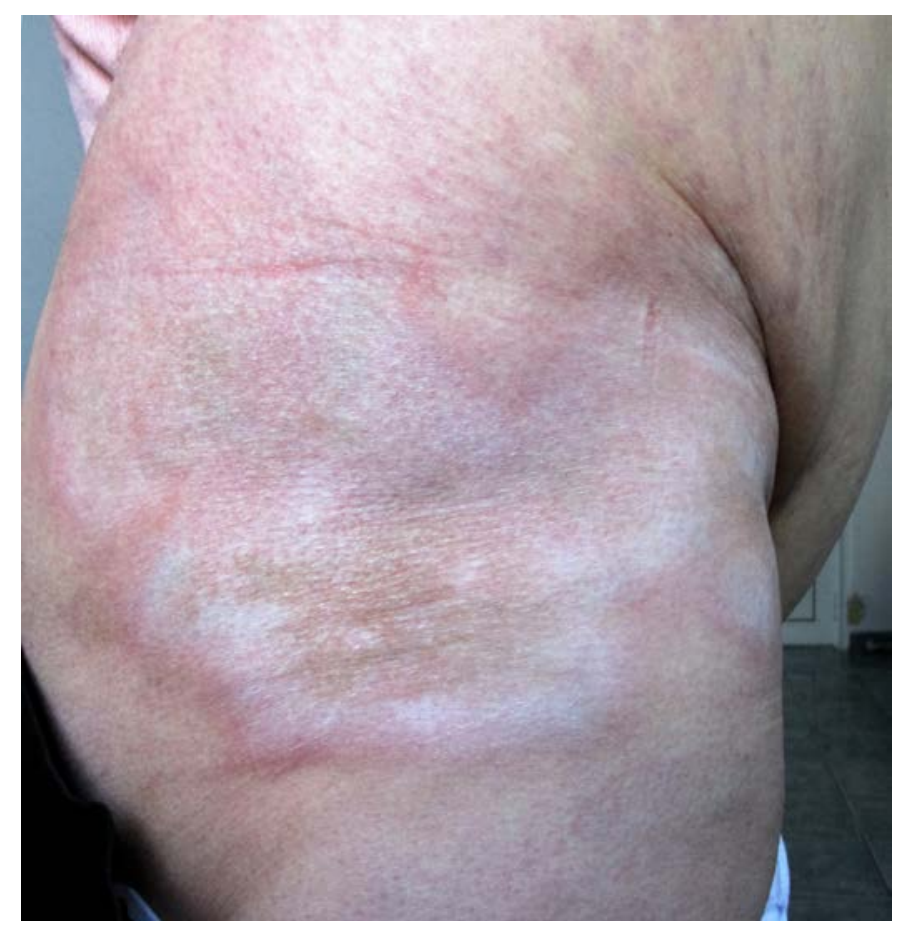

FIGURE 1. Well-circumscribed indurated plaque with a central ivory-white area surrounded by a lilac ring. 

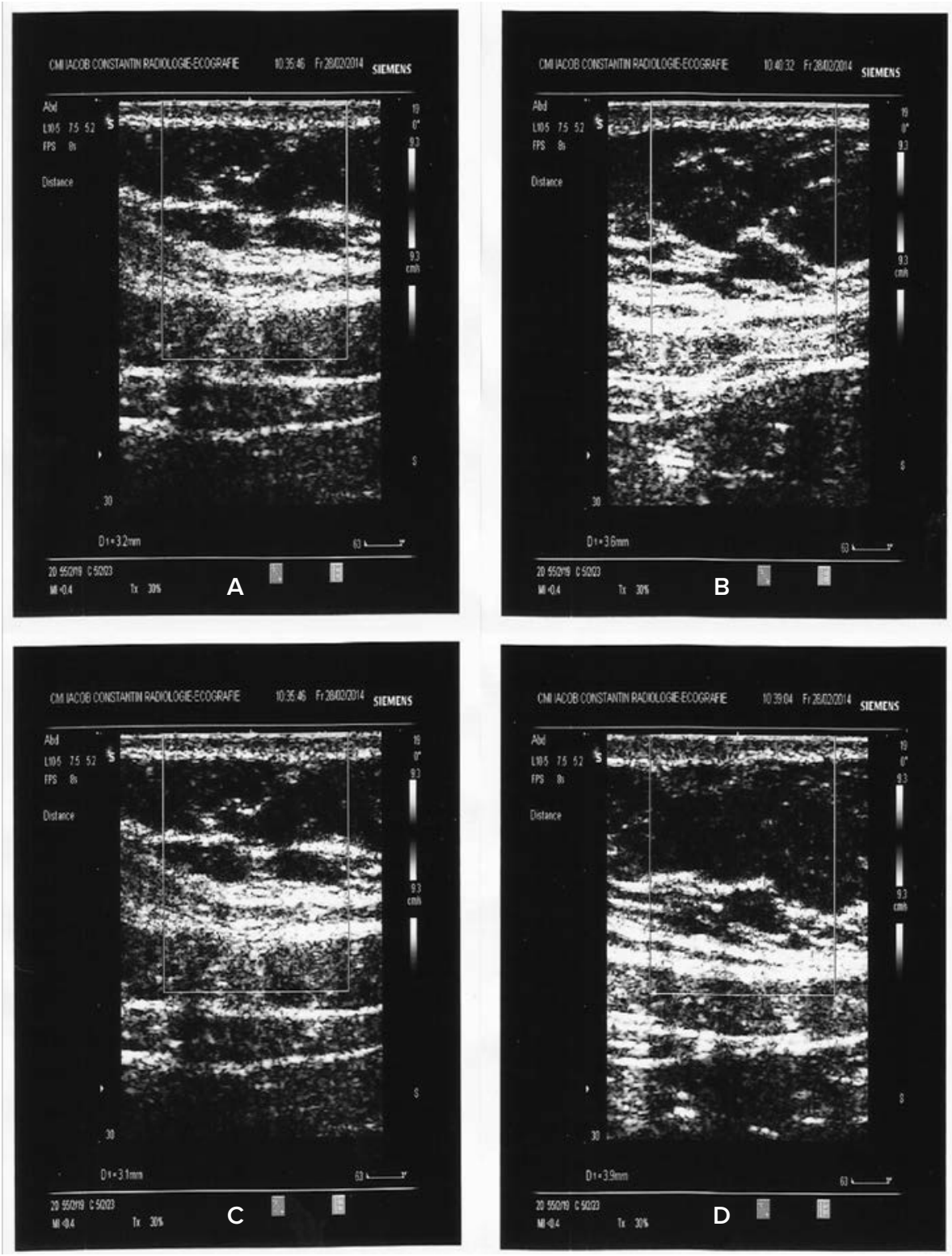

FIGURE 2. Ultrasound images: the process limited to muscle; irregular thickening (3.2 to $3.6 \mathrm{~mm}$ ) in the affected area compared to normal surrounding areas

response to treatment by dynamically measuring the skin thickness reported to the mRSS clinical score (modified Rodnan Skin Score). ${ }^{6}$ A good response to treatment is accompanied by a decrease of dermal and subcutis thickness observed by skin ultrasound.

Similar findings were observed in the present case: skin thickness measured by ultrasonography $(18 \mathrm{MHz})$ correlated with the clinical evolution of morphea. Ultrasound confirmed the localized modifications in the cutis and subcutis, guided the biopsy and turned out to be a tool for evaluating the treatment. ${ }^{7}$

Skin ultrasound is an inexpensive, noninvasive, accessible method. A well-trained radiologist using high-fre- quency ultrasound may be of great help in the examination and follow-up of patients with morphea. High frequency (18 MHz) could be useful for: identification of localized morphea; guided skin biopsy; quantification of skin thickness; evaluating the severity activity by measuring total echogenicity, hypodermis echogenicity, and deep tissue layer vascularity; monitoring the response to treatment by dynamically measuring skin thickness.

\section{CONCLUSIONS}

The ultrasound technique may be of use in choosing skin areas that would yield a positive biopsy specimen, can as- 
sess the size and extension of the lesions and be an objective parameter of patient follow-up.

\section{CONFLICT OF INTEREST}

Nothing to declare.

\section{REFERENCES}

1. Wortsman X. Ultrasound in dermatology: why, how, and when? Semin Ultrasound CT MR. 2013;34(3):177-195.
2. Wortsman $X$, Wortsman J. Ultrasound accuracy in the diagnosis of skin and soft-tissue lesions. AJR Am J Roentgenol. 2015;204(2):W220.

3. Li SC, Liebling MS, Haines KA, Weiss JE, Prann A. Initial evaluation of an ultrasound measure for assessing the activity of skin lesions in juvenile localized scleroderma. Arthritis Care Res (Hoboken). 2011;63(5):735-742.

4. Wortsman $X$, Wortsman J. Clinical usefulness of variable frequency ultrasound in localized lesions of the skin. J Am Acad Dermatol. 2010;62(2):247-256.

5. Wortsman $X$, Vergara $P$, Castro $A$, et al. Ultrasound as predictor of histologic subtypes linked to recurrence in basal cell carcinoma of the skin. J Eur Acad Dermato/ Venereol. 2015;29(4):702-707.

6. Porta F, Kaloudi O, Garzitto A, et al. High frequency ultrasound can detect improvement of lesions in juvenile localized scleroderma. Mod Rheumatol. 2014;24(5):869-873.

7. Wortsman $X$. Common applications of dermatologic sonography. J Ultrasound Med. 2012;31(1):97-111. 